\title{
Chlamydia Trachomatis Infection in High-Risk Human Papillomavirus Based on Cervical Cytology Specimen
}

\author{
Soracha Sangpichai ${ }^{1}$, Natcha Patarapadungkit ${ }^{1 *}$, Chamsai Pientong ${ }^{2}$, Tipaya \\ Ekalaksananan $^{2}$, Surachat Chaiwiriyakul ${ }^{1}$, Ratchaneekorn Thongbor ${ }^{1}$, \\ Phannatorn Sirivech $^{1}$, Porntip Jangsiriwitayakorn ${ }^{1}$, Tippawan Triamwittayanon ${ }^{1}$
}

\begin{abstract}
Objective: High-risk human papillomavirus (HR HPV) was associated with the development of cervical cancer. Asymptomatic Chlamydia trachomatis (C. trachomatis) infection is the most common bacterial, sexually-transmitted infection. This study aimed to investigate the association of $C$. trachomatis in positive HR HPV and the cytological results from liquid-based cytology (LBC). Methods: 150 residual LBC specimens were collected; all of which had undergone cytology and HPV testing by Cobas. The samples were established as $C$. trachomatis using real-time PCR (RT-PCR) with Cryptic F/Cryptic R primers. Results: Of 150 positive HPV findings, the most common $(72.7 \%$, 109/150) were the 12 other HR HPVs (viz., 31, 33, 35, 39, 45, 51, 52, 56, 58, 59, 66, and 68). The cervical cytology of those positive HR HPVs were mostly negative $(70.0 \%, 105 / 150)$. The $C$. trachomatis infections in positive HR HPV were $16 \%(24 / 150)$ HPV. The analysis of the abnormal cytology revealed that $41.6 \%$ had $C$. trachomatis coinfection (C. trachomatis and HPV infection) viz., LSIL (20.8\%), HSIL (12.5\%), and ASC-US (8.3\%). A comparison with positive HPV without $C$. trachomatis co-infection revealed that the highest prevalence was for LSIL, ASC-US, and HSIL (11.1\%, 10.3\%, and 6.4\%, respectively). There was no difference between the abnormalities and negative cervical cytology with negative and positive $C$. trachomatis co-infection in HR HPV positive $(p=0.174)$. Conclusion: C. trachomatis infection was not significantly associated HR-HPV and abnormal cytology. This study confirms the increasing rate of $C$. trachomatis infection in asymptomatic women so routine screening for these infections has been suggested to (a) prevent complications such as the chronic pelvic pain associated with prolong infection and (b) reduce sexual transmission of the infection.
\end{abstract}

Keywords: Chlamydia trachomatis- human papillomavirus- cytology

Asian Pac J Cancer Prev, 20 (12), 3843-3847

\section{Introduction}

Human papillomavirus (HPV) infection in women are usually asymptomatic and most clear spontaneously within 1-2 years (Jenkins, 2007). Persistent infection with various high-risk types of HPV (HR HPV) often progresses to invasive cancer. HPV DNA can be detected in more than $99 \%$ of cervical cancer, of which $70 \%$ are caused by HR HPV16 and 18 (Douglas, 2016). HPV16 was the most common type found in cervical carcinoma in all regions. The other associated risk factors for developing cervical cancer are first coitus age, multiple sexual partners, cigarette smoking, sexually-transmitted disease (STD), immunosuppression, oral contraceptive use, and co-infection with other sexually-transmitted infection (STI) (Jhingran et al., 2014).

Chlamydia trachomatis (C. trachomatis) is the most common bacterial STI worldwide with more than 1.4 million infections in the United States alone (Geisler, 2016). C. trachomatis affects $7.8 \%$ of women with symptomatic lower genital tract infection as detected by cyclohexamide-treated McCoy cells (Rugpao et al., 1993). Among asymptomatic Thai males, 7.4\% were positive by PCR for C. trachomatis in urine (Jiamton et al., 2019). Persons, especially women, with asymptomatic chlamydial infection are at high risk of spreading the pathogens to their partners and themselves suffering serious reproductive morbidity (i.e., to the upper genital tract leading to salpingitis, pelvic inflammatory diseases (PID), ectopic pregnancy, and infertility). Men infected with $C$. trachomatis are often diagnosed as having non-gonococcal urethritis (NGU) and complications may cause epididymitis or orchitis in the upper ascending genital tract.

Several studies have suggested that $C$. trachomatis is a co-factor with HPV in the development of cervical cancer 
(Silins et al., 2005; Jensen et al., 2014). C. trachomatis can moreover establish asymptomatic, persistent infections by several mechanisms, including antibiotic resistance, immune evasion, and apoptosis (Chumduri et al., 2013). In addition, $C$. trachomatis is like other intracellular pathogens that can cause substantial changes in gene expression and protein production in the host at the transcriptional, translational, and post-translational levels. Whether the interaction between HPV and C. trachomatis infection will result in development of cervical cancer remains unclear (Elwell et al., 2016). The current study aimed to investigate the prevalence of $C$. trachomatis in persons positive and at high-risk of HPV (HR HPV) infection based on liquid-based cytology.

\section{Material and Methods}

\section{Specimen collection and preparation}

The study protocol was reviewed and approved by the Ethics Committee of Khon Kaen University, Thailand (HE 591453). We collected 150 residual, routine, positive HPV DNA specimens based on liquid-based cytology (LBC) in ThinPrep ${ }^{\circledR}$ between November, 2016 and November, 2018 using the Cobas $^{\circledR} 4800$ system. The data comprised the individual results for HPV 16 and 18 and the pooled results for the 12 other HR HPV genotypes (viz., 31, 33, $35,39,45,51,52,56,58,59,66$, and 68) processed at the Cytology Unit, Department of Pathology, Faculty of Medicine, Khon Kaen University. The cytological results were categorized following the 2014 Bethesda System as (a) negative for an intraepithelial lesion or malignant (NILM), (b) atypical squamous cells of undetermined significance (ASCUS), (c) low-grade squamous intraepithelial lesion (LSIL), (d) atypical squamous cells that cannot be ruled out as high-grade squamous intraepithelial lesion (ASC-H), (e) high-grade squamous intraepithelial lesion (HSIL), or (f) squamous cell carcinoma (SCC) (Nayar and Wilbur, 2015).

\section{DNA extraction}

The scraped cervical cells were washed twice with phosphate buffer saline (PBS). The cells were lysed in 200 $\mu 1$ of lysis buffer ( $10 \mathrm{mM}$ Tris $\mathrm{HCl}, 0.1 \mathrm{mM}$ EDTA pH 7.5, $1 \% \mathrm{SDS}$ and $0.5 \mathrm{M} \mathrm{NaCl}$ ), to which $15 \mu 1$ of proteinase $\mathrm{K}$ was added. This was then mixed by vortex until clear then incubated at $60^{\circ} \mathrm{C}$ for $30 \mathrm{~min}$. The protein was precipitated by addition of protein precipitation buffer (5 M potassium acetate, $11.5 \mathrm{ml}$ of glacial acetic acid and $28.5 \mathrm{ml}$ of distill water, $\mathrm{pH}$ 5.5): the mixture was centrifuged at 13,500 rpm for $5 \mathrm{~min}$ at $4^{\circ} \mathrm{C}$. The DNA was precipitated with an equal volume of isopropanol and collected by centrifugation at $13,500 \mathrm{rpm}$ for $5 \mathrm{~min}$ at $25^{\circ} \mathrm{C}$ and washed with $70 \%$ ethanol. The DNA pellet was air-dried for $15-30 \mathrm{~min}$ then re-suspended in $40 \mu \mathrm{l}$ of distilled water and stored at $-20^{\circ} \mathrm{C}$ until used.

\section{C. trachomatis DNA detection}

The quality of extracted DNA was checked by amplification of a housekeeping gene (Glyceraldehyde 3-phosphate dehydrogenase; GAPDH). The extracted DNA was determined using GAPDH gene detection. C. trachomatis DNA was detected using SYBR real-time PCR (RT PCR) with Cryptic F/Cryptic R primers. The primers were applied for a Chlamydia cryptic plasmid investigation, which amplifies the $71 \mathrm{bp}$ DNA fragment between the open reading frame 1 and 2 (Jaton et al., 2006). The real-time master mix PCR for the cryptic plasmid region (amplifying ABI 7500 fast, Applied Biosystems) contained $5.0 \mu \mathrm{l}$ of SYBR (SsoAdvancedTM Universal SYBR ${ }^{\circledR}$ Green Supermix), $0.3 \mu 1$ of each primer, $2.4 \mu \mathrm{l}$ of distilled water, and $2 \mu \mathrm{l}$ of DNA sample. Amplification was performed by a pre-warming step at $50^{\circ} \mathrm{C}$ for $2 \mathrm{~min}$, followed by denaturing at $95^{\circ} \mathrm{C}$ for $10 \mathrm{sec}, 45$ cycles at $95^{\circ} \mathrm{C}$ for $15 \mathrm{sec}$, and $60^{\circ} \mathrm{C}$ for $1 \mathrm{~min}$.

\section{Statistical analysis}

The chi-square test was used to analyze the correlation between HPV and C. trachomatis DNA detection. A $p$-value $<0.05$ was considered significant. All statistical tests were performed using STATA version 10.

\section{Results}

Genotype of HPV by cobas ${ }^{\circledR} 4800$ system

The 150 positive HPV testing by Cobas $^{\circledR} 4800$ system revealed 10.7\% (16/150) HPV 16, 8.6\% (13/150) HPV 18, $72.7 \%(109 / 150) 12$ other HR HPV $(31,33,35,39,45$, $51,52,56,58,59,66$, and 68$), 4.7 \%$ (7/150) 12 other HR

\section{HPV testing by $\operatorname{cobas}^{\circledast} 4800$ system}

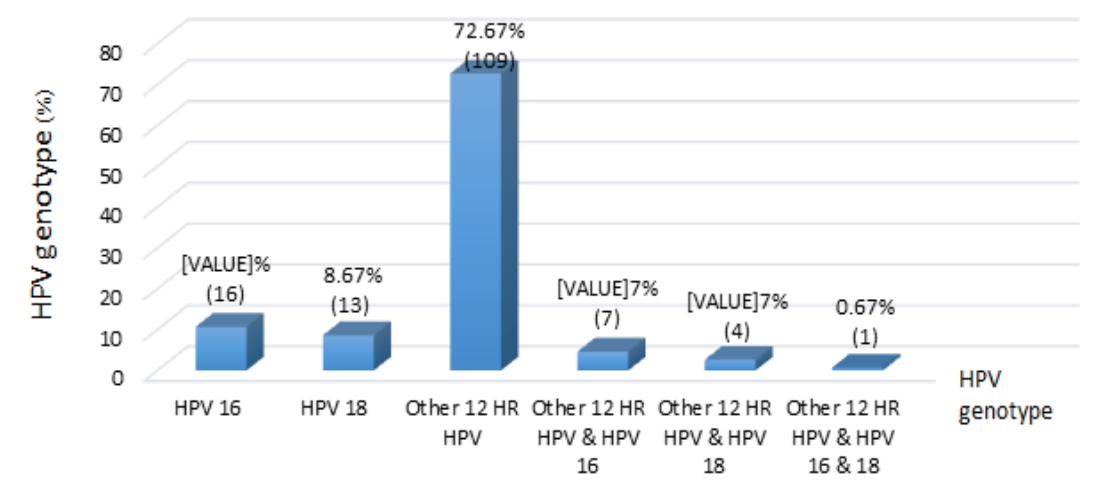

Figure 1. Frequency of 150 Positive HPV Genotype by cobas ${ }^{\circledR} 4800$ System 
Table 1. Comparison of the Frequency Distributions of Cytological Diagnosis and HPV Typing

\begin{tabular}{lcccc}
\hline Positive HPV typing by cobas ${ }^{\circledR} 4800$ system & \multicolumn{3}{c}{ Cytological diagnosis (n=150) } \\
& Negative (\%) & ASC-US (\%) & LSIL (\%) & HSIL (\%) \\
\hline Other 12 HR HPV & $80(76.19)$ & $12(80)$ & $11(57.89)$ & $6(54.55)$ \\
HPV 16 & $11(10.47)$ & $2(13.33)$ & $2(10.53)$ & $1(9.09)$ \\
HPV 18 & $7(6.67)$ & $1(6.67)$ & $3(15.79)$ & $2(18.18)$ \\
Other 12 HR HPV, HPV 16 & $4(3.81)$ & 0 & $2(10.53)$ & $1(9.09)$ \\
Other 12 HR HPV, HPV 18 & $2(1.9)$ & 0 & $1(5.2)$ & $1(9.09)$ \\
Other 12 HR HPV, HPV 16, 18 & $1(0.95)$ & 0 & 0 & 0 \\
Total ( $\mathrm{n}=150)$ & $105(70)$ & $15(10)$ & $19(12.67)$ & $11(7.33)$ \\
\hline
\end{tabular}

HPV plus HPV 16, 2.7\% (4/150) 12 other HR HPV plus HPV 16, and $0.7 \%(1 / 150) 12$ other HR HPV plus HPV 16 and 18 (Figure 1).

\section{Cytological results in positive HR HPV}

The cytological morphology has been reported as NILM, ASCUS, LSIL, and HSIL by the Cytology Unit (Figure 2). The 150 positive HPVs comprised 70.0\% (105/150) negatives, $10.0 \%$ (15/150) ASC-US, $12.7 \%$ $(19 / 150)$ LSIL, and $7.3 \%$ (11/150) HSIL. The negative cytologies found included $76.2 \%(80 / 105)$ of the 12 other HR HPVs, followed by $10.5 \%$ (11/105) of HPV 16 and $6.7 \%(7 / 105)$ of HPV 18 . In addition, there were the 12 other HRs, HPV 16, and/or HPV. In the abnormal cytological group, the 12 other HR HPVs included ASC-US, LSIL, and HSIL (Table 1).

\section{Chlamydia cryptic plasmid detection}

The positive HPV testing detected the chlamydia cryptic plasmid DNA by real-time PCR using the Cryptic-F/Cryptic-R primers. DNA fragments were located between ORF1 and ORF2 of C. trachomatis. The melting temperature $(\mathrm{Tm})$ of the cryptic plasmid

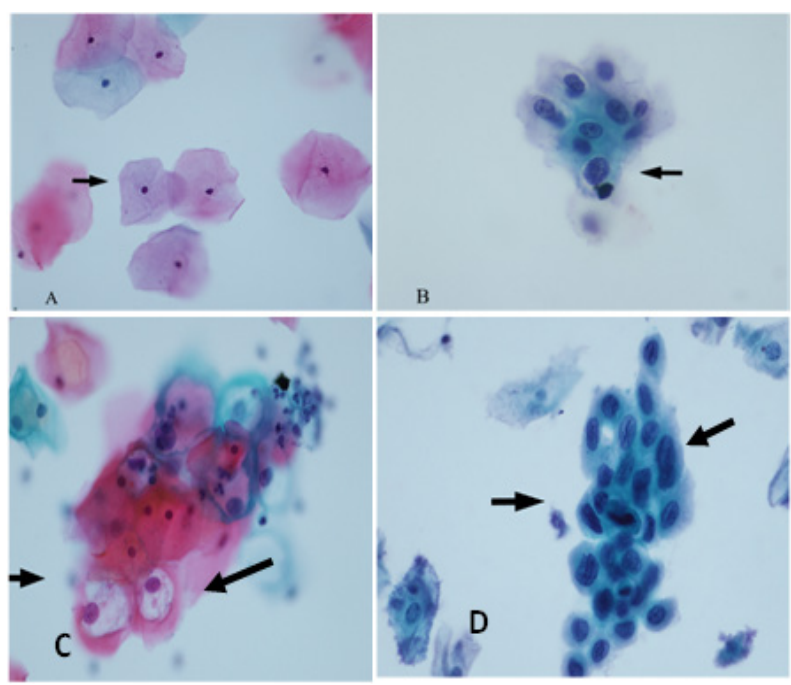

Figure 2. Cytological Samples Classified into Three Groups According to the 2001 Bethesda System. A, negative for intraepithelial lesion or malignancy within normal limits; B, atypical squamous cells of undetermined significance (ASCUS); C, low-grade squamous intraepithelial lesion (LSIL); D, high-grade squamous intraepithelial lesion(HSIL) by Papanicolaou stain $(x 400)$. was determined using Cryptic-F/Cryptic-R real-time PCR at between 79 and $83^{\circ} \mathrm{C}$. The optimal temperature per the melting curve was $\operatorname{Tm} 81{ }^{\circ} \mathrm{C}$. The $71 \mathrm{bp}$ of the PCR products were tested using $2 \%$ agarose gel electrophoresis stained with ethidium bromide (Figure 3). The C. trachomatis infections found included $16 \%$ (24/150) HPV of which the 12 other HR HPV genotypes were the most frequent $(66.7 \%)(16 / 24)$ positive for C. trachomatis while HPV 18 was not found (Table 2).

\section{Association between cytological results and C. trachomatis} infection

We observed an association between abnormal cervical cytology and C. trachomatis in the positive HR HPV. The analysis of $41.6 \%(10 / 24)$ of abnormal cytology (ASC-US, LSIL and HSIL) revealed the highest prevalence of C. trachomatis co-infection in LSIL, followed by HSIL,

Table 2. Frequency of C. trachomatis Detection by SYBR Green Real Time PCR of Each HPV Genotype by cobas $₫ 4800$ System

\begin{tabular}{lc}
\hline 150 HPV genotype & $\begin{array}{c}24(16.0 \%) \text { Positive } \\
\text { C. trachomatis }\end{array}$ \\
\hline HPV 16 & $3(12.5 \%)$ \\
HPV 18 & $0(0 \%)$ \\
Other 12 HR HPV & $16(66.6 \%)$ \\
Other 12 HR HPV \& HPV 16 & $3(12.5 \%)$ \\
Other 12 HR HPV \& HPV 18 & $1(4.2 \%)$ \\
Other 12 HR HPV \& HPV 16 \& 18 & $1(4.2 \%)$ \\
\hline
\end{tabular}

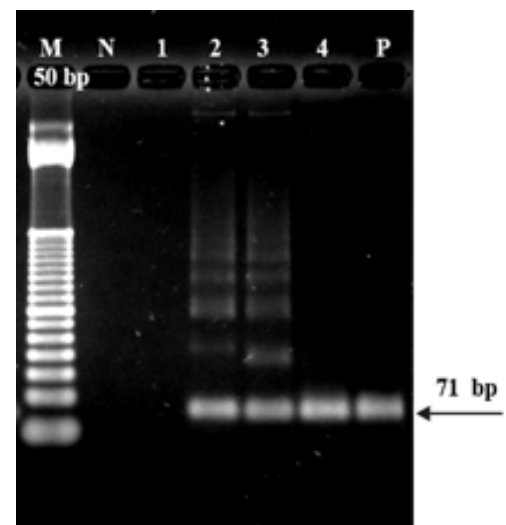

Figure 3. PCR Products of $C$. trachomatis DNA by Cryptic-F/Cryptic-R primers were separated on $2 \%$ agarose gel electrophoresis; M, DNA markers; P, positive control, and 1-4 = samples 
Table 3. Association between Cytology and C. trachomatis Infection in Positive HR HPV

\begin{tabular}{|c|c|c|c|c|c|}
\hline \multirow[t]{2}{*}{ Cytology diagnosis } & \multicolumn{2}{|c|}{ Positive HPV $(n=150)$} & \multirow[t]{2}{*}{ OR $(95 \% \mathrm{CI})$} & \multirow[t]{2}{*}{ Total } & \multirow[t]{2}{*}{ p-value } \\
\hline & Positive C. trachomatis $(n=24)$ & Negative C. trachomatis $(n=126)$ & & & \\
\hline Negative & $14(58.3 \%)$ & $91(72.2 \%)$ & & 105 & 0.174 \\
\hline ASC-US & $2(8.3 \%)$ & $13(10.3 \%)$ & $1.00(0.1-5.2)$ & 15 & \\
\hline LSIL & $5(20.8 \%)$ & $14(11.1 \%)$ & $2.32(0.6-8.2)$ & 19 & \\
\hline HSIL & $3(12.5 \%)$ & $8(6.4 \%)$ & $2.62(0.4-12.8)$ & 11 & \\
\hline Total & 24 & 126 & & 150 & \\
\hline
\end{tabular}

and ASC-US (20.8\%, 12.5\%, and 8.3\%, respectively). The comparison to positive HPV without $C$. trachomatis co-infection revealed that the highest prevalence was for LSIL, followed by ASC-US, and HSIL (11.1\%, 10.3\%, and $6.4 \%$, respectively). The $72.2 \%(91 / 126)$ negative cervical cytology without $C$. trachomatis co-infection was higher than the $58.3 \%(14 / 24)$ C. trachomatis co-infection. There was no significant difference between the abnormalities (ASC-US, LSIL, and HSIL) and negative cervical cytology results of $C$. trachomatis co-infection in positive HR HPV cases $(p=0.174)$. Interestingly, the highest OR was for HSIL, 2.62 (0.4-12.8), followed by LSIL 2.32 (0.6-8.2) and ASCUS 1.00 (0.1-5.2) (Table 3).

\section{Discussion}

Sexually-transmitted viral infections of HPV in the genital tract are common. HPV infection may have transient symptoms, and spontaneous clearance occurs in $80 \%$ of cases as the virus is flushed by the host immune system without any cell changes. In approximately $20 \%$ of women, HPV infection may be persistent and later evolve into cervical cancer in up to $10 \%$ of cases (Abreu et al., 2012; Wohlmeister et al., 2016). Actually, the most important viral factor is the type of HPV. HR HPV genotypes may infect the epithelium persistently, inducing progression and contributing to carcinogenesis. Studies have shown that the most common HR HPV detected in carcinoma cases are HPV 16, 18, 31, 33, 39, and 45 (Muñoz 2000; Kraus et al., 2006; Parkin et al., 2008; Wohlmeister et al., 2016a). Kaliff et al., ( 2018) carried out a study and found that HPV 16 was the most prevalent genotype, followed by HPV 18: the two are the main genotypes detected in squamous cell carcinoma (Oliveira-Silva et al., 2011; Wohlmeister et al., 2016). The 12 other high-risk HPV genotypes (viz., HPV 31, $33,35,39,45,51,52,56,58,59,66$, and 68) were highly prevalent in the present study; more in fact than HPV 16 or HPV 18.

The prevalence of C. trachomatis among asymptomatic women positive for HR HPV in the current study was $16 \%$. The prevalence of genital chlamydia infection in the current study was higher than in previous studies. Thongkrajai et al., (1999) reported a respective prevalence of $C$. trachomatis using the ELISA method of $6.8 \%$, $5.2 \%$, and $6.7 \%$ in rural northeast Thai women attending antenatal, postpartum, and family planning clinics. The prevalence of $C$. trachomatis was investigated using multiplex PCR among students from the northern region of Thailand: the prevalence was between $3.2 \%$ to
$7.5 \%$ (Whitehead et al., 2008). In Hanoi, Vietnam, the prevalence by ELISA technique of lower genital tract C. trachomatis infection was $3.8 \%$ in asymptomatic women attending maternal and child health and family planning clinics (Anh et al., 2003). By comparison, the prevalence of C. trachomatis infection in the United States was $1.7 \%$ (Torrone et al., 2014). In Iran, nested polymerase chain reaction (PCR) used to test the residual fluids of Pap smears: $12.5 \%$ were positive for $C$. trachomatis infection (Javanmard et al., 2018). Differences in study populations and methodologies used for $C$. trachomatis detection yield a wide variation in prevalence rates. The prevalence also varies by age and race/ethnicity. Common co-infections are often asymptomatic, so healthcare providers should routinely screen sexually active women and provide prompt treatment for infected persons (Torrone et al., 2014).

Safaeian et al., (2010) C. trachomatis infection affects HPV persistence and progression to cervical pre-malignancy by a causal disruption of the epithelial tissue. De Paula et al., (2007) reported that although a significant association was found for HPV infection and the precursor lesions of cervical cancer, it was not possible to establish a significant association between these lesions and C. trachomatis co-infection. In the current study, no significance was found between the abnormalities and negative cervical cytology with $C$. trachomatis in HR HPV positive co-infection $(\mathrm{p}=0.174)$. The highest OR presented in HSIL (2.62 [0.4-12.8]) followed by the LSIL and ASCUS groups (2.32 [0.6-8.2] and 1.00 [0.1-5.2]) (Table 3 ). The small number of cases of abnormal cytology in HR HPV make it difficult to confirm whether there are significant differences among the different types of $C$. trachomatis infections.

In conclusion, there were no significantly associated risk factors between $C$. trachomatis infection and HR-HPV infection. The abnormalities of cervical premalignancy are thus not likely associated with C. trachomatis in HR HPV co-infection. Results from the current study confirmed the observation of increasing C. trachomatis infection among asymptomatic women. Routine screening and appropriate treatment for these infections should be performed to prevent complications such as (a) chronic pelvic pain in women, (b) prolonged C. trachomatis infection, and (c) reduced spreading to sexual partners.

\section{Acknowledgements}

The authors thank (a) the patients and their families for their participation; (b) staff in the cytology lab for 
their assistance; and, (c) Mr. Bryan Roderick Hamman for assistance with the English-language presentation of the manuscript under the aegis of the Publication Clinic, Research Affairs, Faculty of Medicine.

\section{Funding Statement}

Research funding included grants from the Faculty of Medicine, and the Graduate School, Khon Kaen University, Thailand (Grant Number IN61218).

\section{References}

Abreu AL, Souza RP, Gimenes F, Consolaro ME (2012). A review of methods for detect human Papillomavirus infection. Virology, 9, 262-70.

Anh PK, Khanh NT, Ha DT, et al (2003). Prevalence of lower genital tract infection among women attending maternal and child health and family planning clinics in Hanoi, Vietnam. Southeast Asian J Trop Med Public Health, 34, 367-73.

Chumduri C, Gurumurthy RK, Zadora PK, Mi Y, Meyer TF (2013). Chlamydia infection promotes host DNA damage and proliferation but impairs the DNA damage response. Cell Host Microbe, 13, 746-58.

Douglas JM (2016). Papillomavirus. In 'Goldman-Cecil Medicine' Goldman L and Schafer AI. Elsevier/Saunders, Philadelphia, 25th ed., pp. 2219-2223.e3.

Elwell C, Mirrashidi K, Engel J (2016). Chlamydia cell biology and pathogenesis. Nat Rev Microbiol, 14, 385-400.

Geisler WM (2016). Diseases Caused by Chlamydiae. In 'Goldman-Cecil Medicine' Goldman L and Schafer AI. Elsevier/Saunders, Philadelphia, 25th ed., pp. 2007-2013.e2.

Jaton K, Bille J, Greub G (2006). A novel real-time PCR to detect Chlamydia trachomatis in first-void urine or genital swabs. $J$ Med Microbiol, 55, 1667-74.

Javanmard D, Behravan M, Ghannadkafi M, et al (2018). Detection of Chlamydia trachomatis in Pap Smear samples from South Khorasan Province of Iran. Int J Fertil Steril, 12, 31-6.

Jenkins D (2007). Histopathology and cytopathology of cervical cancer. Dis Markers, 23, 199-212.

Jensen KE, Thomsen LT, Schmiedel S, et al (2014). Chlamydia trachomatis and risk of cervical intraepithelial neoplasia grade 3 or worse in women with persistent human papillomavirus infection: a cohort study. Sex Transm Infect, 90, 550-5.

Jhingran A, Russell AH, Seiden MV, et al (2014). Cancers of the Cervix, Vulva, and Vagina. In 'Abeloff's clinical oncology'. John NE, James AO, Michael KB, James DH and Joel TE, Elsevier, Churchill Livingstone, Philadelphia. 5th ed. pp 1534-74.e8.

Jiamton S, Leeyaphan C, Chanyachailert P, Surawan T, Omcharoen V (2019). Prevalence of positive PCR assay for Neisseria gonorrhoeae and Chlamydia trachomatis among Asymptomatic Thai Males Attending STD Clinic at Thailand's Tertiary Referral Center. J Med Assoc Thai, 102, 911-7.

Kaliff M, Sorbe B, Mordhorst LB, et al (2018). Findings of multiple HPV genotypes in cervical carcinoma are associated with poor cancer-specific survival in a Swedish cohort of cervical cancer primarily treated with radiotherapy. Oncotarget, 9, 18786-96.

Kraus I, Molden T, Holm R, et al (2006). Presence of E6 and E7 mRNA from human papillomavirus types 16, 18, 31, 33 , and 45 in the majority of cervical carcinomas. J Clin Microbiol, 44, 1310-7.

Muñoz N (2000). Human papillomavirus and cancer: the epidemiological evidence. J Clin Virol, 19, 1-5.

Nayar R, Wilbur DC (2015). The Bethesda System for Reporting Cervical Cytology. In' Comprehensive Cytopathology'. Marluce B and Wilbur DC, Philadelphia, 4th ed., pp 70-81. e2.

Oliveira-Silva M, Lordello CX, Zardo LM, Bonvicino CR, Moreira MA (2011). Human Papillomavirus in Brazilian women with and without cervical lesions. Virology, 8, 4-9.

Parkin DM, Almonte M, Bruni L, et al (2008). Burden and trends of type-specific human papillomavirus infections and related diseases in the latin america and Caribbean region. Vaccine, 26, 1-15.

de Paula FDF, Fernandes AP, Carmo BB do, et al (2007). Molecular detection of Chlamydia trachomatis and HPV infections in cervical samples with normal and abnormal cytopathological findings. Diagn Cytopathol, 35, 198-202.

Rugpao S, Sirirungsi W, Vannareumol P, et al (1993). Isolation of Chlamydia trachomatis among women with symptoms of lower genital tract infection. J Med Assoc Thai, 76, 475-81.

Robial R, Longatto-Filho A, Roteli-Martins CM, et al (2017). Frequency of Chlamydia trachomatis infection in cervical intraepithelial lesions and the status of cytological p16/Ki-67 dual-staining. Infect Agent Cancer, 12, 3-9.

Safaeian M, Quint K, Schiffman M, et al (2010). Chlamydia trachomatis and risk of prevalent and incident cervical premalignancy in a population-based cohort. $J$ Natl Cancer Inst, 102, 1794-804.

Silins I, Ryd W, Strand A, et al (2005). Chlamydia trachomatis infection and persistence of human papillomavirus. Int $J$ Cancer, 116, 110-5.

Thongkrajai P, Thongkrajai E, Pengsaa P, Pakarasang M (1999). The prevalence of Chlamydia trachomatis infection in rural Thai women. Southeast Asian J Trop Med Public Health, 30, 52-7.

Torrone E, Papp J, Weinstock H (2014). Prevalence of Chlamydia trachomatis genital infection among persons aged 14-39 years--United States, 2007-2012. MMWR Morb Mortal Wkly Rep, 63, 834-8.

Whitehead SJ, Leelawiwat W, Jeeyapant S, et al (2008). Increase in Sexual Risk Behavior and Prevalence of Chlamydia trachomatis Among Adolescents in Northern Thailand. Sex Transm Dis, 35, 883-8.

Wohlmeister D, Vianna DRB, Helfer VE, et al (2016). Association of human papillomavirus and Chlamydia trachomatis with intraepithelial alterations in cervix samples. Memórias do Instituto Oswaldo Cruz, 111, 106-13.

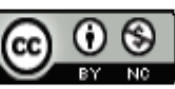

This work is licensed under a Creative Commons AttributionNon Commercial 4.0 International License. 